\title{
INVESTIGACIONES
}

\section{Luche-Mundo: Una metodología para el abordaje responsable y dialógico del TDAH}

\author{
Luche-Mundo: A methodology for a responsible and dialogic ADHD approach \\ Luche-Mundo: Uma metodologia para a abordagem responsável e dialógica do TDAH
}

\author{
Sebastián Claro Tagle \\ Universidad Austral de Chile, Valdivia, Chile. Teléfono: (56) 63072440. \\ Correo electrónico: sebastianclarot@gmail.com
}

\begin{abstract}
RESUMEN
La alta prevalencia del TDAH y la falta de evidencia acerca de tratamientos que tengan un impacto a largo plazo, justifica diseñar y estudiar nuevas formas de abordaje. Luche-Mundo... buscando un tesoro es una metodología creada para abordar colaborativamente este problema. Se funda en un entendimiento de la situación-TDAH. Esto comprende el comportamiento del niño, la percepción de este comportamiento, el entorno y contexto sociocultural. Tras una revisión de las evidencias acerca de los tratamientos del TDAH, se analiza la importancia de la responsabilidad y el diálogo en el diseño de procesos terapéuticos que lo aborden. Además, se esbozan las teorías que sustentan la metodología que se expone, esto es, la teoría del apego, teoría sistémica y aspectos sobre la fantasía.
\end{abstract}

Palabras clave: colaboración, diálogo, fantasía, responsabilidad, TDAH, teoría del apego, teoría sistémica.

\section{ABSTRACT}

The high prevalence of ADHD and the lack of evidence proving a long-term impact of treatments justify the design and study of new strategies for approaching this object. Luche-Mundo... seeking for a treasure is a methodology created for approaching this problem collaboratively. It is based in an understanding of the ADHD-situation. This entails children's behavior, the perception of this behavior, the environment, and the sociocultural context. After a review of the evidence on ADHD treatment, the importance of responsibility and dialogue in the design of therapeutic approaches is analyzed. In addition, attachment theory, systemic theory and aspects related to phantasy are presented as the theories supporting the methodology.

Key words: ADHD, attachment theory, collaboration, dialog, phantasy, responsibility, systemic theory.

\section{RESUMO}

A alta prevalência do TDAH e a falta de evidências sobre os tratamentos que tenham um impacto em longo prazo justificam desenvolver e explorar novas formas de abordagem. Luche-Mundo... buscando um tesouro em uma metodologia criada para abordar, colaborativamente, este problema. Baseia-se na compreensão da situaçãoTDAH, o que compreende o comportamento da criança, a percepção de esse comportamento, o entorno e o contexto sociocultural. Revisam-se as evidências relativas aos tratamentos do TDAH, analisa-se a importância da responsabilidade e do diálogo na concepção de processos terapêuticos que o abordam. Além disso, descrevem-se as teorias que suportam a metodologia definida, isto é, a teoria do apego, teoria sistêmica e aspectos sobre a fantasia.

Palavras chave: colaboração, diálogo, fantasia, responsabilidade, TDAH, teoria do apego, teoria sistêmica. 


\section{INTRODUCCIÓN}

El trastorno por déficit atencional con/sin hiperactividad (TDAH) es el diagnóstico neuropsiquiátrico más utilizado en la infancia. Se caracteriza por manifestaciones de hiperactividad/impulsividad y/o desatención (APA, 2013: 59-60). Dos revisiones de estudios realizados en los cinco continentes concluyen una prevalencia superior al $5 \%$ en la población infantil (Polanczyk et al., 2007; Willcutt, 2012). En Chile, un estudio reciente reporta una prevalencia de $10 \%$ en la población entre 4 y 18 años, alcanzando un 16,4\% en niños hombres entre 4 y 11 años (Vicente et al., 2012). Este diagnóstico concita una importante polémica, enfrentando a quienes afirman que se trata de una enfermedad genuina (Barkley, 2002; Biederman \& Faraone, 2005) y quienes sostienen que es un producto social (Timimi \& Taylor, 2004), un fraude (Baughman, 2006) o un mito (Stolzer, 2009).

La alta prevalencia del TDAH en niños se asocia a un notable aumento en la indicación de psicoestimulantes (Scheffler et al., 2007). No existe evidencia acerca del impacto, en el largo plazo, de este tratamiento (Molina et al., 2009). Por su parte, se han implementado tratamientos distintos al farmacológico, destacando las terapias conductuales con participación de los padres y las terapias realizadas en el contexto escolar (DuPaul \& Weyandt, 2006; Pelham \& Fabiano, 2008). Tampoco estas alternativas terapéuticas han demostrado impacto en el largo plazo y se discute sus ventajas respecto de las terapias farmacológicas (Oord et al., 2008; Pelham et al., 2000).

La prevalencia del TDAH y la falta de evidencia acerca de tratamientos que tengan un impacto en el largo plazo, justifica indagar acerca del abordaje de este diagnóstico y proponer alternativas terapéuticas. En este artículo se expone una perspectiva situacional para entender el TDAH, se revisan las evidencias acerca de los tratamientos estudiados, se exponen principios y teorías que sustentan una metodología para abordar esta entidad, la que es posteriormente descrita.

\section{DE NIÑO-PROBLEMA A SITUACIÓN-PROBLEMA}

El trastorno por déficit atencional con/sin hiperactividad (TDAH) es un diagnóstico neuropsiquiátrico incluido en el Diagnostic and Statistical Manual of Mental Disorders (DSM). En este Manual se identifica el problema de un individuo, el que suele explicarse a través de modelos neurocognitivos (Henríquez-Henríquez et al., 2010). Por su parte, algunos investigadores estudian factores culturales, sociales y familiares que se asocian a déficits en el desarrollo neurocognitivo (Lillard \& Peterson, 2011; Smith et al., 2008), como también su asociación con las manifestaciones del TDAH (Christakis et al., 2004; Clarke et al., 2002; Swing et al., 2010; Thorella et al., 2012). La diversidad de factores asociados a las manifestaciones descritas en esta entidad diagnóstica ha llevado a proponer que, para entender esta entidad, se requiere integrar conocimientos provenientes de las ciencias de la salud y de las ciencias sociales (Singh, 2008). Coherente con ello, se recurre a modelos de los procesos de salud/enfermedad (Engel, 1977) o del desarrollo humano (Bronfenbrenner, 1979), en los cuales se considera lo biológico, psicológico y social (Cooper, 2008; Singh, 2002; Stolzer, 2005).

Avanzando un paso más, sostenemos que el entendimiento del fenómeno al cual alude el TDAH debe partir de la concepción de una situación-problema y no de un niño-problema 
(Claro, 2011). En esta situación-problema un niño manifiesta un comportamiento, el cual es percibido y valorado por uno o más adultos como hiperactivo/impulsivo y/o desatento; esto se da en un entorno como el comedor de la casa, la sala de clase, entre otros, y en un contexto sociocultural. En consecuencia, al analizar el problema por el cual se sospecha un TDAH, el foco no se pone en el niño, sino en las situaciones donde se percibe el comportamiento disfuncional del niño. Acerca de estas situaciones, se analizarán variables biológicas, psicológicas y sociales que puedan estar favoreciendo que el comportamiento de un niño sea percibido como hiperactivo/impulsivo y/o desatento. En el análisis de la situación-TDAH será importante reconocer, desde aquellas condiciones socio-históricas que han llevado a la definición de un diagnóstico como el TDAH y al modo como circula en la sociedad (Conrad, 2005; Ereshefsky, 2009; Hawthorne, 2010), hasta los ya mencionados factores neurocognitivos presentes en el niño, pasando por factores sociales y psicológicos que puedan afectar su comportamiento, como también, la suceptibilidad de los adultos de percibir hiperactividad/impulsividad y/o desatención en el comportamiento de un niño. El conocimiento de estos factores conforman lo que Basarab Nicolescu, un teórico de la trandisciplina, denomina el "Gran Cuadro" (Nicolescu, 2010). Este Gran Cuadro se construye a partir de conocimientos que provienen de diversas disciplinas y, muy importante, desde la perspectiva del abordaje, es necesario considerar el conocimiento de quienes vivencian la situación-TDAH. Un conocimiento concebido así, estará esencialmente "abierto", es decir, su incompletitud no es un defecto sino una de sus cualidades esenciales, "Knowledge is forever open"1 (Nicolescu, 2010: 26).

El entendimiento que proponemos acerca del TDAH es el de una situación donde se manifiesta el comportamiento de un niño, la que es percibida en un entorno y contexto sociocultural dado. Este entendimiento, por su propia esencia, no se puede completar, y va conformando un Gran Cuadro. Éste se construye con conocimientos provenientes de diversas fuentes, destacando el conocimiento de quienes vivencian la situación. Para que este Gran Cuadro sea posible, se necesita de una participación responsable de los niños, familiares, profesionales de la salud y educación, como también el despliegue de un diálogo donde las distintas perspectivas del conocimiento se inscriban sin imponerse. Qué significa dicha responsabilidad y diálogo, fundamentales en la metodología que describiremos más adelante, lo abordaremos después de realizar una revisión general de las evidencias acerca de los tratamientos del TDAH.

\section{EL CONOCIMIENTO SOBRE EL ABORDAJE DEL TDAH}

La modalidad terapéutica más estudiada acerca del TDAH es la farmacoterapia (Hawthorne, 2010). No existe evidencia concluyente acerca de los efectos adversos de esta modalidad terapéutica (Clavenna \& Bonati, 2014; Hechtman \& Greenfield, 2003). En el corto plazo, los efectos adversos más ampliamente reconocidos son: cefalea, dolor abdominal, inapetencia e insomnio (Clavenna \& Bonati, 2014). En algunas revisiones sistemáticas se concluye la eficacia de los psicoestimulantes en el corto plazo para mejorar las funciones cognitivas (Coghill et al., 2014), optimizar el desempeño y comportamiento académico (Prasad et al., 2013), entre otros. Por su parte, no existen estudios que concluyan la eficacia

\footnotetext{
“El conocimiento está, por siempre, abierto” (Traducción del autor).
} 
de la farmacoterapia en el largo plazo. El Estudio del Tratamiento Multimodal en Niños con TDAH (MTA), la investigación más citada acerca del tratamiento de esta entidad, es un estudio clínico randomizado, en el que se intervino por 14 meses a 4 cohortes de niños -intervención farmacológica (IF), terapia conductual (TC), IF + TC, tratamiento en la comunidad- entre 7 y 9,9 años, diagnosticados con TDAH combinado. Al cabo de este período, quienes realizaron la investigación concluyeron que la IF logró los mejores resultados, los que fueron optimizados al agregar la terapia conductual solo en algunas mediciones (MTA, 1999). Otro grupo de investigadores concluyó que la TC sola obtuvo mejores resultados en algunas variables que IF + TC (Pelham et al., 2000). También se publicaron críticas a la metodología utilizada en el estudio (Breggin, 2000). Por su parte, después de 6 y 8 años de iniciada la intervención, no se observaron diferencias entre los grupos; además, comparado con un grupo control, reclutado a los 2 años de iniciado el estudio, los grupos intervenidos durante 14 meses obtuvieron resultados más negativos (Molina et al., 2009). De otra parte, algunos estudios han concluido que la farmacoterapia en el corto plazo sería más costo-efectiva que el no-tratamiento y que las terapias conductuales (Wu et al., 2012), aunque otros estudios relativizan estos hallazgos, afirmando que las conclusiones acerca de las diferencias podrían revertirse o, al menos, estrecharse según la elección de los indicadores de efectividad (Pelham \& Fabiano, 2008).

Otra modalidad terapéutica ampliamente estudiada, aunque significativamente menos que la farmacoterapia, es la terapia conductual. El estudio y la diseminación de esta modalidad terapéutica son más complicados que en el caso de la farmacoterapia. Son varios los obstáculos para demostrar la efectividad de estos tratamientos: el costo de los estudios, la estandarización de las intervenciones, los resultados a medir, entre otros. Aun así, se han recolectado evidencias que demostrarían su efectividad. En un meta-análisis del año 2008 , se concluye que la farmacoterapia y los tratamientos psicosociales tendrían un efecto positivo en la reducción del comportamiento- $\mathrm{TDAH}^{2}$, aunque el efecto de los primeros sería mayor (Oord et al., 2008). En la mayoría de las intervenciones estudiadas, un componente importante está orientado a modificar el comportamiento de los adultos responsables, aunque éste no sea su objetivo terapéutico primario (Daley et al., 2014). En relación a esto, no habría evidencia acerca de la superioridad de intervenciones específicamente orientadas a padres de niños diagnosticados con TDAH, respecto de intervenciones más generales. El efecto positivo se reflejaría en la crianza, en el auto-concepto de los adultos responsables y en la disminución de los problemas de conducta de los niños.

En una revisión de la literatura hasta septiembre del 2006 (Pelham \& Fabiano, 2008) se recomiendan tres tipos de intervenciones psicosociales basados en la evidencia: entrenamiento conductual de padres, intervenciones conductuales con pares y manejo conductual en la sala de clase. Por su parte, aunque sea debatible la ventaja de costoefectividad de la farmacoterapia, no lo es su menor costo de implementación. Por esta razón, algunos investigadores han estudiado modalidades de terapia conductual que pudieran ser menos costosas, como las intervenciones parentales auto-dirigidas. En un meta-análisis reciente, se concluye que estas intervenciones son comparables a aquellas dirigidas por terapeutas, en variables como la percepción de los padres acerca de los cambios en las conductas de los niños (Tarver et al., 2014). Otros investigadores critican las variables que

\footnotetext{
Por comportamiento-TDAH nos referimos a la manifestación de hiperactividad/impulsividad y/o desatención, de acuerdo a la descripción sintomática que se realiza en el DSM (APA, 2013: 59-60).
} 
se busca impactar y se miden en estos estudios, tanto en aquellos que evalúan los efectos de la farmacoterapia como la terapia conductual, proponiendo dirigir las intervenciones hacia la modificación de otros aspectos, como aquellos que se presentan en la relación diádica entre el niño y el adulto responsable (Heriot et al., 2001).

En el contexto escolar, se ha implementado un número importante de intervenciones para niños diagnosticados con TDAH. Estas intervenciones son de tipo conductual, académica y social. Entre ellas, son las primeras las que han mostrado evidencias en su efectividad, provocando cambios positivos en la conducta de los niños y en el tiempo dedicado a la realización de actividades académicas (DuPaul \& Weyandt, 2006).

A partir de esta revisión, se concluye que se disponen de terapias tanto farmacológicas como conductuales, con probada efectividad en disminuir las manifestaciones del comportamiento-TDAH, como también para mejorar el funcionamiento académico y conductual del niño. Sin embargo, no se ha podido demostrar un efecto importante en el funcionamiento social y no se ha demostrado que este efecto perdure en el tiempo. Entonces, resulta necesario atender a lo que concluyen los investigadores del MTA, después de 8 años de iniciado su estudio:

There is a practical need to pursue further research to develop and deliver more effective sustainable interventions, and to shift the emphasis in the field from reliance on ADHD symptoms as the key outcome of treatment to include measurement of impairments that bring families in for treatment and that are likely to mediate adulthood functioning ${ }^{3}$ (Molina et al., 2009: 498).

Más importante que modificar determinadas manifestaciones del niño, los cambios que pueden proyectarse en el tiempo serán aquellos que se den en la familia y, agregamos, otros grupos humanos donde el niño se desarrolla (grupo-curso, grupo-barrio, grupoamigos). Esto no justifica rechazar el uso de fármacos, aunque las modificaciones en las manifestaciones del niño que estos provocan pueden conducir a cambios en otras dimensiones y personas (Heriot et al., 2001), pero implica necesariamente que, en caso de utilizarlos, esta sola intervención será insuficiente. Otro aspecto que importará al momento de diseñar una metodología terapéutica para abordar la situación-TDAH, será el costo y, en relación a ello, su replicabilidad en los ámbitos públicos de salud, educación y comunitarios. Esto, en la mayoría de los países del mundo y para la mayor parte de la población mundial, resulta ser una variable determinante para su acceso.

Un propósito general y fundamental de Luche-Mundo, la metodología que se describirá más adelante, es la movilización de los recursos terapéuticos del niño, de la familia, de los profesionales de salud y educación, en una dinámica colaborativa. No se trata de recursos terapéuticos para abordar un diagnóstico neuropsiquiátrico, cada vez más conocido, del que se ha recolectado evidencia para determinar cuáles tratamientos son más efectivos. Más bien son recursos terapéuticos que se fundan en la responsabilidad y el diálogo posibles en virtud del encuentro y del proceso que realiza un grupo de personas, a propósito de la situación-TDAH. En este encuentro, los profesionales de la salud y educación tendrán la responsabilidad de compartir los conocimientos alcanzados en sus respectivas disciplinas,

"existe una necesidad práctica de investigar, para desarrollar e implementar intervenciones más efectivas y con mayor impacto en el tiempo, y cambiar el énfasis en este campo, de considerar los síntomas TDAH como el resultado principal del tratamiento, incluyendo mediciones de las deficiencias que llevan a las familias a consultar y que probablemente actúan como mediadores en el funcionamiento del adulto" (Traducción del autor). 
como los descritos previamente acerca de la efectividad de los tratamientos farmacológicos y psicosociales. Por su parte, estos conocimientos serán reconfigurados a propósito de aquellos que los niños, sus familiares y otros profesionales serán responsables de compartir, en la constante y nunca completa construcción del Gran Cuadro del TDAH. La relevancia de la responsabilidad y del diálogo no se limita al proceso diagnóstico y de construcción de este Cuadro en una determinada circunstancia, sino que se extiende al proceso terapéutico y a la creación de condiciones para que los efectos de éste se proyecten en el tiempo.

\section{LA RESPONSABILIDAD EN EL DIAGNÓSTICO Y COMPORTAMIENTO-TDAH}

En la década de los 70, se popularizó el concepto de "medicalización" de la sociedad o de la vida. Ivan Illich (1975) denunciaba que, "El monopolio médico y paramédico sobre la metodología y la tecnología de la higiene es un ejemplo notorio del uso político indebido que se hace de los progresos científicos en provecho de la industria y no del ser humano" (p. 9). Más adelante, en Némesis Médica, advierte que "A medida que la institución médica se apropia de la administración del sufrimiento, disminuye mi responsabilidad por mí y tu sufrimiento" (p. 117). En los últimos dos decenios, se pasó de una visión acerca de la medicalización como un proceso "negativo" promovido principalmente por los médicos y la industria farmacéutica, a la descripción de un proceso más complejo en el cual participan quienes son diagnosticados y utilizan los fármacos (Fainzang, 2013; Williams \& Calnan, 1996) y se reconocen "buenas" y "malas" formas de medicalización (Parens, 2013).

El TDAH es un diagnóstico que ha sido estudiado en la perspectiva de los procesos sociales de medicalización (Conrad \& Schneider, 1992; Rafalovich, 2013). Esto vuelve relevante el tema de la responsabilidad, aunque ahora se inscribe en un discurso algo diferente del afirmado por Illich. En la actualidad, cabrá preguntar acerca de cómo debe asumirse la responsabilidad ante el sufrimiento que pudiera asociarse a un comportamiento percibido como hiperactivo/impulsivo y/o desatento. ¿Son los padres y profesores responsables, en primer lugar, de sospechar el diagnóstico precozmente y consultar a un profesional de la salud? Y si no es esto, ¿cómo entender su responsabilidad? ¿Qué responsabilidad le cabe a los padres y profesores ante el sufrimiento que el niño manifiesta, asociado a su comportamiento? Esto plantea una pregunta sustantiva desde la perspectiva del tratamiento del TDAH, más aún, cuando resulta razonable afirmar que los cambios en la conducta de los padres son un mediador potente para lograr transformaciones a largo plazo en las manifestaciones-TDAH (Daley et al., 2014).

A principios del siglo XX, al plantear una posible anormalidad física como causa del "Déficit en el control moral", considerado por algunos investigadores como la primera descripción de un problema semejante al TDAH (Barkley, 2006), George Still se preguntaba, "how far these children are to be held responsible for their misdoings"4 (Still, 1902: 1167). Otros investigadores han analizado las dificultades para determinar la responsabilidad respecto de los comportamientos, al biologizar el comportamiento-TDAH (Bowden, 2014; Tait, 2003; Vehmas, 2011). En las décadas que siguieron a la Segunda Guerra Mundial, algunas teorías influyentes acerca del origen de la psicopatología destacaban el rol de las madres (Bateson, 1972) y, consecuentemente, criterios de una buena crianza. Preocupada

"hasta dónde deben considerarse responsables estos niños de sus malos comportamientos" (Traducción del autor). 
por eso, Stella Chess, investigadora influyente en la concepción del TDAH, preguntaba, "How many mothers (...) will now begin to feel guilty and anxious if they fail to meet this prescription?"5 (1983: 975). Por su parte, investigaciones acerca de discursos de madres y padres de niños diagnosticados con TDAH, realizados en países diversos culturalmente, concluyen que los padres, y especialmente las madres, se sienten culpables por el mal comportamiento de sus hijos (Brunton et al., 2014; Frigerio et al., 2013; Kildea et al., 2011; Klasen, 2000; Malacrida, 2001; Uribe \& Rojas, 2007). En estos estudios se describe que el diagnóstico médico y la concepción biológica del comportamiento-TDAH alivian la culpa. Por eso, Ilina Singh caracteriza el discurso de madres de niños diagnosticados con TDAH como modelo de comportamiento sin-culpable (Singh, 2004). El curso de esta desresponsabilización, sin embargo, se complica: el alivio provocado es de corta duración (Kildea et al., 2011), la responsabilidad de los padres se reconfigura después del diagnóstico, centrándose en la enfermedad del hijo (Brunton et al., 2014; Malacrida, 2001; Singh, 2004; Uribe \& Rojas, 2007), los padres transfieren la responsabilidad a otros (Frigerio et al., 2013), se legitima la experiencia personal y favorece una sensación de poder y agencia respecto del comportamiento del niño (Klasen, 2000). Por su parte, en una investigación sobre los discursos cotidianos acerca del TDAH en Estados Unidos, tanto en medios de comunicación como en conversaciones informales, se concluye que uno de los cinco tópicos más ampliamente abordados es el conflicto entre la causa biológica del comportamiento diagnosticado con TDAH y la culpa moral (Danforth \& Navarro, 2001). Los autores destacan una resistencia a la explicación biológica y al uso que se le da para no asumir la responsabilidad en los comportamientos.

El discurso biomédico del TDAH ha tenido una gran influencia práctica, evidente por el aumento en el uso de psicoestimulantes para modificar el comportamiento-TDAH, en los últimos 25 años. Esto podría explicarse por el efecto que tiene este fármaco en el corto plazo, evidenciable por padres y profesores, y es posible que lo explique el hecho de favorecer que la culpa que sienten los adultos responsables reciba algún tipo de alivio. Sin embargo, esta des-responsabilización sigue un curso que está lejos de poder ser descrito de manera uniforme e inequívoca. Esto podría reflejar una resistencia a lo que Martha Farah plantea a propósito de las posibles consecuencias derivadas del gran conocimiento que se ha alcanzado sobre el funcionamiento neurológico del ser humano: "Such a transformation could reduce us to machines in each other's eyes, mere clockwork devoid of moral agency and moral value" (2012: 587-588). Además, el discurso biomédico, aunque pueda favorecer la desresponsabilización, también se asocia a factores no deseados por las personas y negativos para los procesos terapéuticos. En un meta-análisis de reciente publicación (Kvaale et al, 2013), la explicación biogenética de los problemas de salud mental, junto con reducir el sentimiento de culpa, se asociaba con una perspectiva pesimista respecto del pronóstico del problema. Por su parte, en un estudio acerca del TDAH, en el cual se comparaba la explicación biológica con la psicológica (Lebowitz et al., 2012), la primera favorecía la disminución del sentimiento de culpa y una mayor aceptación social, y también un mayor pesimismo respecto del pronóstico y menores expectativas en las intervenciones psicosociales.

“¿Cuántas madres... se sentirán culpables y ansiosas si no cumplen estas prescripciones?” (Traducción del autor).

"Esa transformación puede reducirnos a máquinas ante la mirada de los otros, simples mecanismos de relojería, carentes de agencia moral y valor moral" (Traducción del autor). 
Aunque el comportamiento de los humanos implique un sustrato biológico, salvo que eso nos reduzca a la condición de "máquinas desprovistas de agencia y valores morales", será necesario considerar qué responsabilidad cabe y cómo desplegarla respecto de los comportamientos. ¿Cómo diseñar procesos donde participen responsablemente el niño y uno o más adultos significativos? ¿Cómo favorecer que los profesionales de salud y/o educación, participando responsablemente, no inhiban la participación de los niños y adultos significativos? Las posibilidades de esto, en una medida importante, dependerán de las relaciones que se establezcan en los procesos terapéuticos. Para analizar y proponer condiciones de dichas relaciones, se analizarán las cualidades del diálogo en los procesos terapéuticos de la situación-TDAH.

\section{EL DIÁLOGO TERAPÉUTICO EN LA SITUACIÓN-TDAH}

En el abordaje de la situación-TDAH es sustantivo el diálogo entre quienes participan del proceso terapéutico, en dos sentidos. Por un lado, éste es condición de que todos los que participen del proceso terapéutico, especialmente los niños y sus familiares, reconozcan que sus vivencias, sus emociones y entendimientos configuran el proceso en curso. $\mathrm{Si}$ el proceso es impuesto por el médico u otro profesional de salud, desde el diagnóstico hasta las indicaciones terapéuticas, sin considerar suficientemente lo excepcional de la experiencia de "este" niño y "esta" familia, la posible agencia de niños y familiares se verá complicada. Entonces, su responsabilidad se restringirá a aceptar el diagnóstico y seguir el tratamiento propuesto. Este tipo de procesos terapéuticos pueden alcanzar resultados efectivos, pero esta falta de agencia de niños y adultos responsables ¿no influirá en que sus efectos no persistan en el tiempo? Al priorizar la evidencia disciplinar por sobre un diálogo donde se implica la vivencia de niños y familiares ¿no se limitan las proyecciones del cambio?

El diálogo es importante por las oportunidades de participación y agencia que implica, y más fundamentalmente, por su estatuto en la existencia humana. La percepción de hiperactividad/impulsividad y/o desatención en el comportamiento de un niño sucede en un entorno y contexto sociocultural, donde se inscriben expectativas respecto del comportamiento humano. A este comportamiento se le atribuyen funciones, se implican trayectorias diagnósticas y terapéuticas, justificadas por presupuestos acerca del origen de los comportamientos humanos y de su posibilidad de transformación. Estas trayectorias están condicionadas por la concepción que se despliega acerca del ser humano.

En la perspectiva que se desarrolla acá se atribuye al Otro o a lo Social la condición de fundamento de su singularidad. En ello se sigue a Vigotsky (2001), quien afirma que "La verdadera dirección del proceso de desarrollo del pensamiento del niño no va de lo individual a lo socializado, sino de lo social a lo individual" (p. 59). En contra de Piaget, quien afirma que el lenguaje egocéntrico surge como consecuencia de la insuficiente socialización del lenguaje inicialmente individual, en la opinión del psicólogo ruso "surge de la insuficiente individualización del lenguaje inicialmente social, de su incipiente separación y diferenciación, de su inespecificidad" (p. 314). De acuerdo a esta concepción, lo social no es un epifenómeno en el desarrollo humano, sino un constituyente de su individualidad, fundamento en la formación de su pensamiento y lenguaje. No acontece un desarrollo individual para luego encontrarse con otros y socializar, sino que en lo social se 
va desarrollando y diferenciando el individuo. Mijaíl Bajtín (2000) describe este proceso de la siguiente forma, "Todo lo que se refiere a mi persona, comenzando por mi nombre, llega a mí por boca de otros (la madre), con su entonación, dentro de su tono emocional y volitivo. Al principio, tomo conciencia de mí mismo a través de los otros: de ellos obtengo palabras, formas, tonalidad para la formación de una noción primordial acerca de mí mismo" (p. 161). El ser humano, para el filósofo ruso, no posee un territorio soberano, no hay tal cosa como un interior que ha germinado a espaldas de las miradas de los otros, el ser humano "siempre y por completo se encuentra en la frontera; al mirar en su interior, mira a los ojos del otro, o bien a través de los ojos del otro" (p. 163). Para Bajtín, el diálogo constituye la existencia humana:

La única forma adecuada de la expresión verbal de una auténtica vida humana es el diálogo inconcluso. La vida es dialógica por su naturaleza. Vivir quiere decir participar en un diálogo: preguntar, poner atención, responder, estar de acuerdo. En este diálogo, el hombre completo toma parte con toda su vida: ojos, labios, manos, alma, espíritu, el cuerpo entero, los actos. Su ser entero se le va en la palabra, que se introduce en el tejido dialógico de la vida de los hombres, en el simposio universal (2000: 165).

La sustancia del proceso terapéutico de la situación-TDAH coincide con este "diálogo inconcluso". En este proceso no participará el niño-TDAH, el niño-disfuncional; es imprescindible que participe el niño completo, con sus sonrisas, sus rostros de pregunta, sus posturas en movimiento, sus emociones confusas. De igual forma, sus padres no serán los padres a desculpabilizar ni a responsabilizar por el cumplimiento de unas indicaciones, no serán los padres-disfuncionales, los padres-abnegados, los padres-sobreprotectores, los padres-ausentes. Es necesario que los niños, los padres y quienes más participen en este proceso, se descubran en los ojos del otro, no objetivados ni exigidos a cumplir un rol, sino en una mirada que se encuentra en la frontera, ese lugar que no me pertenece ni le pertenece al otro, pero que sólo es posible por nosotros, en ese diálogo en el cual participamos.

En Verdad y Método, Hans-Georg Gadamer realiza una descripción fenomenológica de la conversación que resulta significativa a propósito del diálogo terapéutico (Eaton, 1998). En particular, interesan tres aspectos: la unidad de una intención, el lenguaje común y la transformación hacia lo común (Gadamer, 2003). Para Gadamer, "el arte de llevar una conversación, es al mismo tiempo el arte de mirar juntos en la unidad de una intención" (p. 446). A esto se debe aspirar en un proceso terapéutico donde, junto al niño y su familia, participan personas formadas en una o más disciplinas. Esa unidad de intención, por cierto, no puede ser impuesta, y tan sólo queda buscar su consecución en la misma conversación. Por su parte, aunque las formaciones y experiencias de quienes participan del proceso terapéutico sean distintas, aunque los lenguajes y las herramientas sean diversos, el proceso no será posible si no se va desplegando un lenguaje común. Relacionado con esto, Gadamer afirma que "Toda conversación (...) constituye desde sí un lenguaje común (...) algo aparece puesto al medio, y los interlocutores participan de ello y se participan entre sí sobre ello" (p. 457). En la conversación se elabora un lenguaje común, lo que no deriva de un proceso externo, ni tampoco se debe a que los conversadores se adaptan unos a otros, en vez de eso, cada uno va entrando en la conversación en la medida en que se va dando lugar a ese lenguaje común (p. 458). En el curso de la conversación, el acuerdo, "no es un mero exponerse e imponer el propio punto de vista, sino una transformación hacia lo común, 
donde ya no se sigue siendo el que se era" (p.458). Esta transformación a lo común está lejos de implicar una mismidad, una homogenización. Eso no es deseable ni posible. Más bien, mezclando conceptos de Bajtín y Gadamer, se trata de una transformación vueltos hacia la frontera, la que se constituye en el lenguaje común.

No se desconoce que el proceso terapéutico de la situación-TDAH tiene como motivo transformar la situación del niño. Por eso, en el diálogo terapéutico no es equivalente la transformación de cualquiera de los participantes. Estas importan en la medida en que afectan la situación-TDAH. Pero, por un lado, si se despliega el diálogo inconcluso, donde se expresa la auténtica vida humana, cualquiera será transformado. Y, por otro lado, siendo lo Social, el Otro, el diálogo, constituyente de la persona, la pura transformación individual carece de sentido. Si no se producen transformaciones en el contexto del niño, en sus relaciones con los adultos, en el entorno donde suele ser percibido como hiperactivo/ impulsivo y/o desatento, ¿cabrá tener expectativas de que sus transformaciones individuales perduren? En la perspectiva de la situación-TDAH, el niño no sólo deja de ser el centro del problema, también deja de ser el centro de la transformación. Esto no implica que el motivo del proceso no sea la transformación del niño, pero se reconoce que ello sólo será posible si sucede la transformación de su situación. En términos de Bajtín, no es el interior del niño el que se busca transformar, sino el despliegue de sus fronteras.

\section{TEORÍA DEL PROCESO TERAPÉUTICO: APEGO, SISTEMA Y FANTASÍA}

Expuestos los principios del proceso terapéutico de la situación-TDAH, se hará un esbozo sobre las perspectivas de lo humano que se propone enfocar en este proceso. Es decir, se responderá la pregunta acerca de qué conocimientos permiten articular un proceso donde el despliegue de la responsabilidad y el diálogo se orienten efectivamente al abordaje de las problemáticas de la situación-TDAH. Este conocimiento debe estar vinculado a dichas problemáticas y, a la vez, favorecer el despliegue de la responsabilidad y el diálogo. A continuación, veremos cómo la teoría del apego, la teoría sistémica y aspectos teóricos de la fantasía aportan en este proceso.

En la teoría del apego se destaca la importancia de la figura de apego en el desarrollo del ser humano (Bowlby, 1989). Esta teoría remite a la conducta de apego, determinada biológicamente y tan vital como la reproducción, abrigo y alimentación, contribuyendo a la supervivencia del individuo y de su descendencia. Un concepto básico de esta teoría es el de base segura. Una figura de apego positiva se convierte en una base segura, favoreciendo las posibilidades de exploración del niño, quien aprende a confiar que, ante peligros o inseguridad, podrá retornar a la seguridad de su base o figura de apego. En el desarrollo de la conducta de apego se da un proceso de internalización de "modelos operantes que un niño construye de su madre y de los modos en que ella se comunica y se comporta con él, y un modelo comparable de su padre, junto con los modelos complementarios de sí mismo en interacción con cada uno" (p. 151). Esos modelos operantes se afirman como estructuras cognitivas influyentes. Varios estudios han mostrado la asociación entre apego inseguro, particularmente de tipo desorganizado, y el comportamiento-TDAH (Clarke et al., 2002; Roskam et al., 2014). Aunque no se puede afirmar que todo niño que presenta un comportamiento-TDAH presenta un apego inseguro, las investigaciones justifican considerar el tipo de apego del niño, como un aspecto importante al momento de evaluar 
y abordar este problema. Eso sí, no hay evidencias de que intervenciones centradas en modificar aspectos del apego, como pudieran ser los modelos operantes mencionados más arriba, tengan un impacto positivo en las manifestaciones-TDAH. En consideración a esto, y sin descartar que futuras investigaciones aporten evidencia positiva respecto de estas intervenciones, vale ampliar la perspectiva del apego con la perspectiva sistémica, esto es, ampliar una teoría enfocada en la relación del niño con su figura de apego, con una teoría que considera un sistema más amplio de relaciones y circunstancias.

La integración de las teorías del apego y sistémica-familiar se inició en la década de los 90. Entonces, se crearon conceptos como el de base familiar segura, indicando una red familiar que posibilita a sus miembros la seguridad suficiente para explorar (ByngHall, 1995). Destacando la relevancia de las relaciones significativas del niño, se agrega la importancia de considerar que ellas se dan en un contexto donde participan otras personas. Por su parte, sin desconocer la excepcional relevancia que puedan tener los vínculos afectivos en el sistema familiar, especialmente con los padres, se amplía el análisis a otros vínculos afectivos que se desarrollan a lo largo de la vida (Ainsworth, 1989). Además, la teoría sistémica enfatiza los recursos existentes en el contexto familiar y comunitario (Sydow, 2002). Mientras la primera realiza aportes significativos sobre las consecuencias de ciertas circunstancias ocurridas en el pasado, la teoría sistémica ofrece una perspectiva más orientada al presente y el futuro, enfatizando los recursos disponibles para un mejor desarrollo.

Para un adecuado abordaje de la situación-TDAH, junto con el apego y el sistema donde se desarrolla el niño, relevamos la importancia de la fantasía. Este término, que se sobrepone al de imaginación, se entenderá como la creencia en entidades o procesos que no se explicarían por lo que consideramos los principios de la naturaleza (Woolley, 1997). Esta definición es amplia y ambigua, pero suficiente en este contexto. En el ámbito de la psicología, la fantasía ha tenido una gran importancia, especialmente en el psicoanálisis, destacado en la obra de una influyente psicoanalista infantil, como fue Melanie Klein (Segal, 2003). Por su parte, el rol de la fantasía en el desarrollo infantil genera controversia, la que se vuelve manifiesta al analizar las posturas respecto de fenómenos frecuentes como son los amigos imaginarios (Bonne et al., 1999; Gleason \& Hohmann, 2006; Hoff, 2005). Otra controversia se refiere a las diferencias relativas a la fantasía entre niños y adultos. Suele afirmarse una distinción cualitativa fundamental, aunque varias investigaciones muestran que estas diferencias no serían tales. En los resultados de dichas investigaciones no se rechazan las diferencias entre la fantasía de niños y adultos, las que estarían determinadas por motivos culturales, de conocimiento y adquisición de conceptos fundamentales, disposición a aceptar lo fantástico junto a lo real, capacidad para detectar inconsistencias en las creencias y para descartar por completo la realidad de algo que más bien parece fantástico (Woolley, 1997). Lo que se destaca es, por un lado, que los adultos despliegan una fantasía que puede llegar a ser muy importante en sus vidas y, por otro lado, desde muy pequeños, los niños son capaces de distinguir entre fantasía y realidad.

Cuatro motivos justifican incluir la fantasía en los procesos orientados al abordaje de la situación-TDAH:

a) La fantasía permite a los niños y a los adultos imaginar otras posibilidades de lo real. Aquello que en el mundo real simplemente es, en la fantasía se transforma. Esto adquiere especial relevancia si la fantasía es co-creada, particularmente en conjunto con los padres 
o en un contexto psicoterapéutico con el terapeuta (Bonovitz, 2004). El desafío es que esa fantasía se inscriba en un proceso que conduzca a la transformación de la realidad, en un sentido deseado por el niño y sus adultos significativos.

b) La fantasía está presente en la vida de los niños, especialmente en sus juegos. El rol del juego en la infancia está ampliamente documentado y su función principal en el desarrollo del niño es reconocida (Winnicott, 2002). Integrar la fantasía en un formato de juego favorece que el niño pueda expresar su mundo. Según Linaza, en el juego se manifiesta "la forma específica con la que los niños abordan la realidad, sea física, social o intelectual" (Linaza, 2013: 104).

c) Cuidar la fantasía de los niños y participar en ella es relevante en el desarrollo del niño. Para especificar lo que se dice, daremos dos ejemplos: Fonagy y Target (2007) describen una situación en la cual un niño de dos años y medio jugaba con una silla, como si fuera un tanque; cuando su padre le preguntó si aquello con lo que jugaba era una silla o un tanque, el niño la puso en su lugar y se fue. Por su parte, Bonovitz (2010) recuerda un amigo imaginario que vivía en una ciudad distinta a la de él; éste desapareció después de que su padre lo llevara a la estación de tren para que visitara a ese amigo. En estos ejemplos se destaca la fragilidad del mundo fantástico de los niños y el rol de los adultos en su desarrollo o, al menos, en su continuidad. Más complejo es afirmar la importancia de ese desarrollo y qué cualidades conviene que tome. Pero, tomando en cuenta lo señalado anteriormente -sus posibilidades transformativas y la tendencia del niño a la fantasía a través del juego- es planteable que, al menos en alguna medida, será importante que los adultos cuiden y participen del mundo fantástico de los niños.

d) Lo que se llama lenguaje fantástico -también llamado lenguaje creativo o artístico, es decir las comunicaciones cuyo contenido no se explica por lo que consideramos los principios de la naturaleza- tendrá un gran potencial de descubrir aspectos de quien se manifiesta, que de otra forma permanecen en la sombra. Esto será válido para niños y adultos. Este recurso es ampliamente utilizado con niños donde el juego, el dibujo, entre otros, tienen tanto un rol diagnóstico como terapéutico.

De acuerdo a lo desarrollado, se propone un abordaje de la situación-TDAH, donde se consideren los conocimientos acerca del apego, del sistema donde se desarrolla el ser humano y de la fantasía. En lo que sigue, se describirá la metodología Luche-Mundo y la inclusión de estos desarrollos teóricos en su diseño.

\section{LUCHE-MUNDO... DESCUBRIENDO UN TESORO}

Luche-Mundo... buscando un tesoro (LM) es una metodología creada para abordar problemas como la situación-TDAH. Su nombre se debe al juego infantil: luche o rayuela. $\mathrm{Al}$ igual que en este juego, se invita a recorrer casilleros/estaciones. Estas estaciones están en un mundo fantástico llamado Luche-Mundo. Este mundo es habitado por dos personajes: los Luchemunderos. Para viajar se establecen algunas pocas reglas y, principalmente, se invita a relacionarse, imaginar y crear, lo que se promueve a través del contexto fantástico y el envío de cartas. De esta forma, aunque se puede discutir si LM se corresponde con un juego, integra aspectos lúdicos como los listados por Cordier et al. (2009): motivación intrínseca, control interno, libertad para suspender la realidad y la definición de un marco o 
contexto. Esta condición de la metodología es fundamental por lo ya señalado acerca de la fantasía y, de acuerdo con Vigotsky, "play provides a much wider background for changes in needs and in consciousness"7 (Cit. en Baumer \& Radsliff, 2009: 13).

LM se implementa en escuelas, centros de salud u otras organizaciones orientadas al desarrollo de los niños. Uno o dos miembros de la organización facilitan el proceso de 5 a 15 niños, quienes son acompañados, cada uno, por un adulto significativo. Los niños son elegidos por cumplir criterios de la situación-TDAH: ser percibido por uno o más adultos como hiperactivo/impulsivo y/o desatento. El adulto significativo debe vivir con el niño o, al menos, convivir regularmente. El facilitador invita al niño y su acompañante a realizar un viaje por el Luche-Mundo. Al iniciar el viaje, se les entrega una carta enviada por los luchemunderos, quienes dan la bienvenida al Luche-Mundo y cuentan sobre cuatro caminos, cada uno con cuatro estaciones, todos los cuales se dirigen al centro del Luche-Mundo: al Umbral. Los luchemunderos envían una carta cada vez que el niño y su acompañante llegan a una estación - estas cartas las tiene el acompañante, quien decidirá cómo hacerlas llegar para que no se interrumpa la fantasía-. A través de estas cartas invitarán al niño a realizar una actividad con su acompañante y, a veces, con otras personas. En cada estación se utiliza un cuaderno, donde se dibuja, escribe o pega algo, siempre relacionado con la actividad realizada. Terminada la actividad, se dirigirán a la siguiente estación. Una vez recorridas las 16 estaciones, se alcanza el Umbral. Entonces, se realiza una ceremonia donde asisten los niños, sus acompañantes y personas cercanas de ellos. En esta instancia, junto con compartir acerca del viaje, las actividades que más los motivaron, lo que aprendieron, se descubre el tesoro: la vida del niño. Para eso, los acompañantes envuelven una foto del niño en un marco especial y un libro creado a partir del cuaderno que se fue completando a lo largo del viaje; en las últimas páginas de este libro, el acompañante y otras personas cercanas, dan testimonio de por qué la vida del niño es un tesoro para ellos.

Es fundamental que los acompañantes asuman responsablemente el trabajo que se realizará, esto es, con creatividad y cuidando la relación con el niño. Con el objeto de otorgar mejores condiciones para que esta participación se asuma adecuadamente, el facilitador se reúne con ellos antes de iniciar el viaje. En esta instancia se les da a conocer la metodología y se dialoga acerca de cómo implementarla, de tal forma que resulte motivante y significativa para los niños que acompañarán. La cualidad de este diálogo es fundamental para que los adultos reconozcan su agencia responsable en el proceso y se establezca una relación con el o los facilitadores y entre ellos, donde cada cual reconozca su voz en este diálogo. Mientras se viaja por el Luche-Mundo, aproximadamente 16 semanas, los acompañantes, junto con el facilitador, se reúnen periódicamente para dialogar acerca de las oportunidades y dificultades encontradas en el proceso, además de aquello que han descubierto en los niños y aprendido durante este viaje. También se comparten textos donde se tratan temas relativos a la crianza. En estos encuentros resulta sustantivo el diálogo entre los adultos, inspirado por sus experiencias y los significados que les otorgan. Finalizado el viaje, ellos organizarán la ceremonia del Umbral. En general, el diálogo estará orientado a descubrir cómo, a partir de la experiencia que se desarrolla, se despliegan aprendizajes que la trascienden, descubriendo otras oportunidades para los comportamientos y las relaciones, encarnando estos descubrimientos/aprendizajes en hábitos, conductas y acciones diarias, que den testimonio de ello.

\footnotetext{
"El juego ofrece un contexto más amplio para los cambios en las necesidades y en la conciencia" (Traducción del autor).
} 
En la versión creada para abordar la situación-TDAH, los temas de los cuatro caminos son la biografía, los movimientos, la atención y el asombro. En cada estación habita un animal, el que tiene unas características especiales: vivir muchos años, piel muy sensible, vista prodigiosa, gran inteligencia o memoria, entre otros. Las actividades propuestas en las estaciones se relacionan con el tema del camino y con aquello que hace especial al animal que ahí habita. En cada estación, junto con proponer las actividades, los luchemunderos cuentan algo sobre el animal. Además, se le cuenta a los niños que estos animales se encontraron con un Ser que vive en el Umbral, al que los luchemunderos llaman Multicolor. En un sueño, ellos descubrieron que este Ser cambia de formas y colores, fruto de la imaginación de los niños. Por eso, los invitan a imaginar su Multicolor, el que representarán y caracterizarán cuando lleguen al Umbral.

El diseño de la metodología Luche-Mundo... buscando un tesoro se funda en lo desarrollado más arriba. Se enfatizará cómo se inscribe cada uno de los dos principios destacados: responsabilidad y diálogo, y de los tres cuerpos teóricos: apego, sistema y fantasía.

Responsabilidad. Este proceso de abordaje de la situación-TDAH carece de un diseño normativo, es decir, no se pre-definen logros a alcanzar ni es estricta la secuencia a seguir. Si bien se establecen hitos y etapas a cumplir, más se enfatiza cuidar el proceso, particularmente en cómo cada uno de los niños, adultos significativos y facilitadores entienden y despliegan su participación. En cada una de las instancias de encuentro, reuniones de adultos significativos con facilitador, realización de las actividades entre el niño y su acompañante, ceremonia del umbral, es principal que cada participante reconozca su agencia, tanto en la fantasía del viaje, como en las acciones que forman parte de la realidad que se comparte. En este sentido, cada instancia es una oportunidad para descubrir la responsabilidad que me cabe respecto del otro, específicamente en este proceso de viaje por el Luche-Mundo y, a partir de eso, trascendiendo el viaje.

Diálogo. Son varios los conocimientos o saberes importantes en este viaje. No podría existir una disciplina donde se desarrolle un conocimiento que hegemonice el diseño y despliegue del viaje por el Luche-Mundo. La particularidad de cada viaje, de los conocimientos y saberes relevantes que ahí se descubren, está consagrada en el diseño de esta metodología. Cada participante: niño, adulto significativo, facilitador, dispone de un conocimiento insustituible y absolutamente necesario. La sustancia del viaje es el proceso donde las voces de cada participante se descubren en el contexto de las otras voces en un ir hacia esas oportunidades excepcionales, de significados y sentidos, que otorga este diálogo.

Diálogo y responsabilidad. La responsabilidad se manifiesta en el diálogo. Dialogar en la frontera es un acto de plena responsabilidad. Alcanzar dicha frontera solo posible en virtud de mí, pero imposible sin la presencia del otro, es una manifestación plena de responsabilidad con el otro. Lograr este diálogo en la frontera o diálogo responsable es una tarea ardua en nuestros tiempos; $y$, se debe ser cauto: no hay decreto que lo pueda imponer. Permanentemente, a lo largo del viaje, es prioritario estar atento al despliegue de las posibilidades del diálogo, a las cualidades de mi participación, a las condiciones que dispone el otro y, muy especialmente, a los niños, para participar responsablemente del diálogo.

Apego. La unidad relacional del proceso la constituye el niño con su adulto significativo, de preferencia, la figura materna o paterna. Con esto se reconoce el rol destacado e insustituible de las relaciones más significativas del niño. Esto justifica que el facilitador, quien en otros modelos terapéuticos forma parte de lo que se está llamando la unidad relacional del proceso, en este caso cumple el rol de facilitar ciertas condiciones para 
que el niño y el adulto significativo puedan explorar otras posibilidades en su relación y, eventualmente, aunque no se tienen evidencias acerca de esto, que se modifique el tipo de apego o, al menos, algunas cualidades de los modelos operantes internalizados por el niño.

Sistema. El Viaje por el Luche-Mundo se inscribe en la vida cotidiana. Algunas actividades se extienden a momentos habituales, como la hora de acostarse, la comida, entre otros, y todas las actividades se proponen como un estímulo para decidir acerca de otras actividades a realizar regularmente. Además, aunque la figura constante del viaje para el niño es el adulto significativo que participa como acompañante, en varias actividades se incluyen otras personas: amigos, familiares, vecinos. A su vez, se invita a que personas cercanas den testimonio de la vida del niño como un tesoro, aparte de quien acompaña en el viaje y en la ceremonia del umbral se propone que participen familiares y amigos del niño. A esto, a propósito del viaje y las experiencias que se realizan, se pueden agregar más instancias de diálogo con profesores y otras personas significativas para el niño. Por lo demás, las experiencias que se van desarrollando en el viaje son posibles a partir de los recursos disponibles en el entorno del niño, especialmente en lo que se refiere a personas, pero también a la naturaleza y recursos materiales. Todo esto da cuenta de la importancia que se le otorga al sistema donde se desarrolla el niño, tanto para entender y abordar sus problemáticas, como para acceder a recursos que le puedan transformar.

Fantasía. El Luche-Mundo es una fantasía. Es habitado por personajes fantásticos: los luchemunderos. Ellos se comunican con los niños a través de cartas. El Multicolor es un ser fantástico a quien lo transforma la imaginación de los niños. En esta metodología, el contexto del viaje es fantástico y se privilegia una co-creación por parte del niño y su acompañante. Al proyectar esta fantasía, importa considerar las cualidades antes señaladas, que la hacen importante en esta metodología: sus posibilidades transformativas, su relevancia en los juegos de los niños, la fragilidad del mundo fantástico de los niños y su potencial reflejo de lo que, en su ausencia, queda oculto. En esta metodología la fantasía importa en el diálogo, responsablemente compartida, lo que implica vivir y recrear lo fantástico, no sólo a propósito de mí, sino de igual forma, en virtud del otro. En esto, la participación de niños y adultos no es equivalente, correspondiéndole a estos últimos un despliegue más cuidadoso.

Apego, sistema y fantasía. La relación de apego, con la consecuente internalización de modelos operantes, es aquello que se espera transformar a lo largo del proceso de viaje por el Luche-Mundo y, posteriormente, en virtud de los cambios inscritos en los hábitos y costumbres del niño y personas significativas. Esto sucede en un sistema donde hay otros influyentes y se dispone de recursos que, por un lado, pueden favorecer esta transformación y, por otro lado, la pueden hacer manifiesta y extender sus alcances. Para potenciar las oportunidades de transformación que se dan en el encuentro entre personas cuya relación es significativa en tanto partes de un sistema, se despliega un mundo de fantasía donde adquiere sentido la transformación, el juego, el cuidado y el descubrimiento del otro.

\section{EPÍLOGO}

Los niños que reciben algún diagnóstico de salud mental han aumentado en los últimos decenios. Asociado a ello, se evidencia un gran incremento en el uso de fármacos para modificar su comportamiento. Sin embargo, en los estudios realizados respecto del 
diagnóstico más utilizado en la neuropsiquiatría infantil, el TDAH, no se ha logrado evidenciar el impacto positivo del tratamiento farmacológico ni de otros tratamientos, en el largo plazo. Al faltar la evidencia no se puede concluir que este impacto no acontezca, pero debe motivar a seguir investigando y buscando nuevas formas para abordar estas problemáticas. Esto se convierte en un mandato para quienes creen que la falta de impacto positivo se debe a algo más que la intensidad, dosis y/o duración de los tratamientos, atribuyéndolo, más bien, a la forma de entender el problema y, muy especialmente, a cómo participan los niños y adultos significativos en la configuración de dicho entendimiento y en el abordaje de los problemas que los afectan.

En este artículo se expuso una metodología para abordar lo que se ha denominado la situación-TDAH, la que se refiere a la percepción de un niño como hiperactivo/impulsivo y/o desatento. Pasar del desafío de abordar el TDAH de un niño a abordar la situaciónTDAH, supone pasar de implementar un tratamiento centrado en el niño, según lo propone una o más disciplinas, a uno que involucre los diversos momentos de la situación-TDAH, en un proceso de conocimiento y abordaje colaborativo. Luche-Mundo... buscando un tesoro es una metodología orientada al despliegue de procesos transformadores de esta situación, partiendo del entorno del niño e involucrando a sus otros significativos.

\section{REFERENCIAS BIBIOGRÁFICAS}

Ainsworth, M. (1989). Attachments beyond infancy. American Psychologist, 44(4), 709-716.

APA. (2013). Diagnostic and statistical manual of mental disorders (5 ed.). Washinton, DC: American Psychiatric Association.

Bajtín, M. (2000). Yo También Soy. Ciudad de México: Alfaguara.

Barkley, R. (2002). International consensus statement on ADHD. Clinical Child and Family Psychology Review, 5(2), 89-111.

Barkley, R. (2006). The relevance of the Still lectures to attention-deficit/hyperactivity disorder: A commentary. Journal of Attention Disorders, 10, 137-140.

Bateson, G. (1972). Steps to an ecology of mind. New York: Ballantine Books.

Baughman, F. A. (2006). The ADHD fraud: How psychiatry makes "patients" of normal children. Victoria, Canada: Trafford Publishing.

Baumer, S., \& Radsliff, K. (2009). Playworlds of children and adults: Cultural perspectives on play pedagogy. Mind, Culture, and Activity, 17(1), 11-13.

Biederman, J., \& Faraone, S.V. (2005). Attention-deficit hyperactivity disorder. Lancet, 366, 237-248.

Bonne, O., Canetti, L., Bachar, E., Kaplan, A., \& Shalev, A. (1999). Childhood imaginary companionship and mental health in adolescence. Child Psychiatry and Human Development, 29(4), 277-286.

Bonovitz, C. (2004). The cocreation of fantasy and the transformation of psychic structure. Psychoanalytic Dialogues, 14(5), 553-580.

Bonovitz, C. (2010). The interpersonalization of fantasy: The linking and de-linking of fantasy and reality. Psychoanalytic Dialogues, 20, 627-641.

Bowden, G. (2014). Disorders of inattention and hyperactivity: The production of responsible subject. History of the Human Sciences, 27(1), 88-107.

Bowlby, J. (1989). Una Base Segura: aplicaciones clínicas de una teoría del apego. Barcelona: Paidos.

Breggin, P. R. (2000). The NIMH multimodal study of treatment for attention-deficit/ 
hyperactivity disorder: A critical analysis. International Journal of Risk \& Safety in Medicine, 13, $15-22$.

Bronfenbrenner, U. (1979). The ecology of human development. Massachusetts, Estados Unidos: Harvard University Press.

Brunton, C., McVittie, C., Ellison, M., \& Willock, J. (2014). Negotiating parental accountability in the face of uncertainty for attention-deficit hyperactivity disorder. Qualitative Health Research, 24(2), 242-253.

Byng-Hall, J. (1995). Creating a secure family base: Some implications of attachment theory for family therapy. Family Process, 34(1), 45-58.

Clarke, L., Ungerer, J., Chahoud, K., Johnson, S., \& Stiefel, I. (2002). Attention Deficit Hyperactivity Disorder is Associated with Attachment Insecurity. Clinical Child Psychology and Psychiatry, 7(2), 179-198.

Claro, S. (2011). Clima escolar y desarrollo integral de niñas y niños. Historia, aprendizajes y proyecciones de una experiencia. Santiago: RIL Editores.

Clavenna, A., \& Bonati, M. (2014). Safety of medicines used for ADHD in children: A review of published prospective clinical trials. Archives of Disease in Childhood, 99, 866-872.

Coghill, D. R., Seth, S., Pedroso, S., Usala, T., Currie, J., \& Gagliano, A. (2014). Effects of methylphenidate on cognitive functions in children and adolescents with Attention-deficit/ hyperactivity disorder: Evidence from a systematic review and a meta-analysis. Biological Psychiatry, 76(8), 603-615.

Conrad, P. (2005). The Shifting Engines of Medicalization. Journal of Health and Social Behavior, 46, 3-14.

Conrad, P., \& Schneider, J. (1992). Deviance and medicalization. From badness to sickness. Philadelphia: Temple University Press.

Cooper, P. (2008). Like alligators bobbing for poodles? A critical discussion of education, ADHD and the biopsychosocial perspective. Journal of Philosophy of Education, 42(3-4), 457-474.

Cordier, R., Bundy, A., Hocking, C., \& Einfeld, S. (2009). A model for play-based intervention for children with ADHD. Australian Occupational Therapy Journal, 56, 332-340.

Chess, S. (1983). Mothers are always the problem - or are they?. Old wine in new bottles. Pediatrics, 71(6), 974-976.

Christakis, D. A., Zimmerman, F. J., DiGiuseppe, D. L., \& McCarty, C. A. (2004). Early television exposure and subsequent attentional problems in children. Pediatrics, 113(4), 708-713.

Daley, D., Oord, S. v. d., Ferrin, M., Danckaerts, M., Doepfner, M., Cortese, S., \& SonugaBarke, E. J. S. (2014). Behavioral interventions in Attention-Deficit/ Hyperactivity Disorder: A meta-analysis of randomized controlled trials across multiple outcome domains. Journal of American Academy of Child and Adolescent Psychiatry, 53(8), 835-847.

Danforth, S., \& Navarro, V. (2001). Hyper talk: Sampling the social construction of ADHD in everyday language. Anthropology \& Education Quarterly, 32(2), 167-190.

DuPaul, G. J., \& Weyandt, L. L. (2006). School-based Intervention for Children with Attention Deficit Hyperactivity Disorder: Effects on academic, social, and behavioural functioning. International Journal of Disability, Development and Education, 53(2), 161-176.

Eaton, J. (1998). Gadamer: Psychotherapy as conversation. European Journal of Psychotherapy \& Counselling, 1(3), 421-433.

Engel, G. L. (1977). The need for a new medical model: A challenge for biomedicine. Science, 196(4286), 129-136.

Ereshefsky, M. (2009). Defining 'health' and 'disease'. Studies in History and Philosophy of Biological and Biomedical Sciences, 40, 221-227.

Fainzang, S. (2013). The other side of medicalization: Self-medicalization and self-medication. Culture, Medicine \& Psychiatry, 37(3), 488-504.

Farah, M. J. (2012). Neuroethics: The ethical, legal, and societal impact of neuroscience. Annual 
Review of Psycholgy, 63, 571-591.

Fonagy, P., \& Target, M. (2007). Playing with reality: IV. A theory of external reality rooted in intersubjectivity. International Journal of Psychoanalysis, 88, 917-937.

Frigerio, A., Montalia, L., \& Fine, M. (2013). Attention deficit/hyperactivity disorder blame game: A study on the positioning of professionals, teachers and parents. Health, 17(6), 584-604.

Gadamer, H.-G. (2003). Verdad y método. Salamanca: Ediciones Sígueme.

Gleason, T. R., \& Hohmann, L. M. (2006). Concepts of real and imaginary friendships in early childhood. Social Development, 15(1), 128-144.

Hawthorne, S. (2010). Embedding values: How science and society jointly valence a concept-the case of ADHD. Studies in History and Philosophy of Biological and Biomedical Sciences, 41, 21-31.

Hechtman, L., \& Greenfield, B. (2003). Long-term use of stimulants in children with attention deficit hyperactivity disorder. Safety, efficacy, and long-term outcome. Pediatr Drugs, 5(12), 787-794.

Henríquez-Henríquez, M., Zamorano-Mendieta, F., Rothhammer-Engel, F., \& Aboitiz, F. (2010). Modelos neurocognitivos para el trastorno por déficit de atención/hiperactividad y sus implicaciones en el reconocimiento de endofenotipos. Revista de Neurología, 50(2), 109-116.

Heriot, S., Evans, I., \& Foster, T. (2001). An interactional approach to intervention research with children diagnosed with ADHD. Journal of Child and Family Studies, 10(3), 287-299.

Hoff, E. V. (2005). Imaginary companions, creativity, and self-image in middle childhood. Creativity Research Journal, 17(2-3), 167-180.

Illich, I. (1975). Némesis médica. La expropiación de la salud. Barcelona: Barral Editores.

Kildea, S., Wright, J., \& Davies, J. (2011). Making sense of ADHD in practice: A stakeholder review. Clinical Child Psychology and Psychiatry, 16(4), 599-619.

Klasen, H. (2000). A name, what's in a name? The medicalization of hyperactivity, revisited. Harvard Review of Psychiatry, 7(6), 334-344.

Kvaale, E. P., Haslam, N., \& Gottdiener, W. H. (2013). The 'side effects' of medicalization: A meta-analytic review of how biogenetic explanations affect stigma. Clinical Psychology Review, 33(6), 782-794.

Lebowitz, M. S., Rosenthal, J. E., \& Ahn, W.-k. (2012). Effects of biological versus psychosocial explanations on stigmatization of children with ADHD. Journal of Attention Disorders. doi: $10.1177 / 1087054712469255$

Lillard, A. S., \& Peterson, J. (2011). The immediate impact of different types of television on young children's executive function. Pediatrics, 128(4). doi: 10.1542/peds.2010-1919

Linaza, J. L. (2013). El juego es un derecho y una necesidad de la infancia. Bordón, 65(1), $103-$ 117.

Malacrida, C. (2001). Motherhood, resistance and attention deficit disorder: Strategies and limits. Canadian Review of Sociology, 38(2), 141-165.

Molina, B., Hinshaw, S., Swanson, J., Arnold, E., Vitiello, B., Jensen, P., \& Group, M. C. (2009). The MTA at 8 years: prospective follow-up of children treated for combined type ADHD in a multisite study. Journal of American Academy of Child and Adolescent Psychiatry, 48(5), 484-500.

MTA. (1999). A 14-month randomized clinical trial of treatment strategies for attention-deficit/ hyperactivity disorder. Archives of General Psychiatry, 56, 1073-1086.

Nicolescu, B. (2010). Methodology of transdisciplinarity - Levels of reality, logic of the included middle and complexity. Transdisciplinary Journal of Engineering \& Science, 1(1), 19-38.

Oord, S. V. d., Prins, P. J. M., Oosterlaan, J., \& Emmelkamp, P. M. G. (2008). Efficacy of methylphenidate, psychosocial treatments and their combination in school-aged children with ADHD: A meta-analysis. Clinical Psychology Review, 28, 783-800.

Parens, E. (2013). On good and bad forms of medicalization. Bioethics, 27(1), 28-35.

Pelham, W., \& Fabiano, G. (2008). Evidence-based psychosocial treatments for Attentiondeficit/hyperactivity disorder. Journal of Clinical Child \& Adolescent Psychology, 37(1), 184-214.

Pelham, W., Gnagy, E., Greiner, A., Hoza, B., Hinshaw, S., Swanson, J., \& McBurnett, K. 
(2000). Behavioral versus behavioral and pharmacological treatment in ADHD. Children attending a summer treatment program. Journal of Abnormal Child Psychology, 28(6), 507-525.

Polanczyk, G., de Lima, M. S., Horta, B. L., Biederman, J., \& Rohde, L. A. (2007). The worldwide prevalence of ADHD: A systematic review and metaregression analysis. The American Journal of Psychiatry, 164(6), 942-948.

Prasad, V., Brogan, E., Mulvaney, C., Grainge, M., Stanton, W., \& Sayal, K. (2013). How effective are drug treatments for children with ADHD at improving on-task behaviour and academic achievement in the school classroom? A systematic review and meta-analysis. European Child \& Adolescent Psychiatry, 22, 203-216.

Rafalovich, A. (2013). Attention deficit-hyperactivity disorder as the medicalization of childhood: Challenges from and for sociology. Sociology Compass, 7(5), 343-354.

Roskam, I., Stievenart, M., Tessier, R., Muntean, A., Escobar, M. J., Santelices, M. P., \& Pierrehumbert, B. (2014). Another way of thinking about ADHD: the predictive role of early attachment deprivation in adolescents' level of symptoms. Social Psychiatry and Psychiatric Epidemioly, 49, 133-144.

Scheffler, R. M., Hinshaw, S. P., Modrek, S., \& Levine, P. (2007). The global market for ADHD medications. Health Aff, 26(2), 450-457.

Segal, H. (2003). Introducción a la obra de Melanie Klein. Buenos Aires: Paidos.

Singh, I. (2002). Biology in context: Social and cultural perspectives on ADHD. Children \& Society, 16, 360-367.

Singh, I. (2004). Doing their jobs: Mothering with Ritalin in a culture of mother-blame. Social Science and Medicine, 59, 1193-1205.

Singh, I. (2008). Beyond polemics: Science and ethics of ADHD. Nature Reviews Neuroscience, 9(12), 957-964.

Smith, P. K., Jostmann, N. B., Galinsky, A. D., \& Dijk, W. W. v. (2008). Lacking power impairs executive functions. Psychological Science, 19(5), 441-447.

Still, G. (1902). The Goulstonian Lectures on some abnormal psychical conditions in children. Lancet, 159(4104), 1163-1168.

Stolzer, J. (2005). ADHD in America: A bioecological analysis. Ethical Human Psychology and Psychiatry, 7(1), 65-75.

Stolzer, J. (2009). Attention Deficit Hyperactivity Disorder: Valid Medical Condition or Culturally Constructed Myth? Ethical Human Psychology and Psychiatry, 11(1), 5-15.

Swing, E. L., Gentile, D. A., Anderson, C. A., \& Walsh, D. A. (2010). Television and video game exposure and the development of attention problems. Pediatrics, 126(2), 214-221.

Tait, G. (2003). Free will, moral responsibility and ADHD. International Journal of Inclusive Education, 7(4), 429-446.

Tarver, J., Daley, D., Lockwood, J., \& Sayal, K. (2014). Are self-directed parenting interventions sufficient for externalising behaviour problems in childhood? A systematic review and meta-analysis. European Child \& Adolescent Psychiatry, 23(12), 1123-1137.

Thorella, L. B., Rydellb, A.-M., \& Bohlin, G. (2012). Parent-child attachment and executive functioning in relation to ADHD symptoms in middle childhood. Attachment \& Human Development, 14(5), 517-532.

Timimi, S., \& Taylor, E. (2004). In debate. ADHD is best understood as a cultural construct. British Journal of Psychiatry, 184, 8-9.

Uribe, C. A., \& Rojas, R. V. (2007). Factores culturales en el trastorno por déficit de atención e hiperactividad: Habla la mamá. Revista Colombiana de Psiquiatría, XXXVI(2), 255-291.

Vehmas, S. (2011). Disability and moral responsibility. Trames, 15(2), 156-167.

Vicente, B., Saldivia, S., De la Barra, F., Melipillán, R., Valdivia, M., \& Kohn, R. (2012). Salud mental infanto-juvenil en Chile y brechas de atención sanitarias. Revista Medica de Chile, 140, $447-$ 457. 
Vigotsky, L. S. (2001). Obras Escogidas - Tomo II. Madrid: A. Machado Libros.

Von Sydow, K. (2002). Systemic attachment theory and therapeutic practice: A proposal. Clinical Psychology and Psychotherapy, 9, 77-90.

Willcutt, E. (2012). The prevalence of DSM-IV Attention-Deficit/Hyperactivity Disorder: A meta-analytic review. Neurotherapeutics, 9, 490-499.

Williams, S. J., \& Calnan, M. (1996). The 'limits' of medicalization?: Modern medicine and the lay populace in 'late' modernity. Social Science \& Medicine, 42(12), 1609-1620.

Winnicott, D. (2002). Realidad y Juego. Barcelona: Gedisa.

Woolley, J. (1997). Thinking about fantasy: Are children fundamentally different thinkers and believers from adults? Child Development, 6(6), 991-1011.

Wu, E., Hodgkins, P., Ben-Hamadi, R., Setyawan, J., Xie, J., Sikirica, V., \& Erder, M. H. (2012). Cost effectiveness of pharmacotherapies for attention-deficit hyperactivity disorder. A systematic literature review. CNS Drugs, 26(7), 581-600. 